\title{
Cortical Metabolism, Acetylcholinesterase Staining and Pathological Changes in Alzheimers Disease
}

\author{
E.G. McGeer, P.L. McGeer, H. Kamo, H. Tago and R. Harrop
}

\begin{abstract}
The local cerebral metabolic rate for glucose (LCMRgl) was determined by positron emission tomography (PET) using the ${ }^{18} \mathrm{~F}$-fluorodeoxyglucose method in a series of Alzheimer patients and normal controls. The LCMRgl declined in the cerebral cortex with age, but the decrement was significantly greater in the clinically diagnosed Alzheimer's cases. Comparison of PET and psychological data indicated that, as the disease progressed clinically, the reduction in cortical LCMRgl and the number of cortical regions involved also increased. Variable regions of cortex were involved in the early stages but the temporal, parietal and frontal regions were most typically affected. One case coming to autopsy showed that the severity of the LCMRgl decline paralleled loss of neurons in the cortex and their replacement with astroglia.

A case of Pick's disease coming to autopsy had shown a different and highly characteristic pattern of cortical metabolic defect. In this case also a poor metabolic rate was associated with extensive gliosis.

Acetylcholinesterase (AChE) staining of the cerebral cortex in elderly normals and Alzheimer's disease cases with a new, highly sensitive method showed that in Alzheimer's disease there was an extensive loss of AChE-positive fibers with senile plaques frequently incorporating AChE-positive fiber debris. AChE staining of the substantia innominata area, where the cells giving rise to these neocortical fibers are presumably located, also showed evidence of degenerating cells and fibers.
\end{abstract}

RÉSUMÉ: Métabolisme cortical, coloration pour la mise en évidence de l'acétylcholinestérase et changements pathologiques dans la maladie d'Alzheimer. Nous avons déterminé la vitesse du métabolisme local du glucose dans le cerveau (VMLgIC) au moyen de la tomographie à émission de positrons (PET), par la méthode utilisant le ${ }^{18} \mathrm{~F}$-fluorodeoxyglucose chez une série de patients atteints de la maladie d'Alzheimer et chez des témoins normaux. La VMLglC diminuait avec l'âge au niveau du cortex cérébral; cependant la diminution était plus grande de façon significative chez les cas portant le diagnostic clinique de maladie d'Alzheimer. La comparaison des données obtenues par la tomographie et l'évaluation psychologique montraient que, avec la progression clinique de la maladie, la diminution de la VMLgIC corticale et le nombre des régions corticales touchées augmentaient également. Des régions variables du cortex étaient atteintes dans les phases précoces de la maladie; cependant, il est tout-à-fait typique de retrouver une atteinte des régions temporales, pariétales et frontales. Un cas, sur, lequel nous avons pratiqué une autopsie, montrait que la sévérité de la diminution de la VMLgIC était en parallèle avec la perte des neurones du cortex et leur remplacement par l'astroglie.

Un cas de maladie de Pick, sur lequel nous avons pratiqué une autopsie, avait montré un tableau de déficit métabolique cortical différent et très caractéristique. Dans ce cas également la lenteur du métabolisme était associée à une gliose importante.

La coloration du cortex cérébral pour la mise en évidence de l'acétylcholinestérase (AChE) chez les vieillards normaux et chez des patients atteints de la maladie d'Alzheimer, au moyen d'une méthode nouvelle très sensible, a montré que, dans la maladie d'Alzheimer, il y a une perte importante de fibres AChE-positives ainsi que des débris de fibres AChE-positifs qui sont souvent incorporés dans les plaques séniles. La coloration pour la mise en évidence de l'AChE au niveau de la substantia innominata, région où sont présumément situées les cellules donnant naissance à ces fibres du néocortex, montrait également des signes de dégénérescence des cellules et des fibres.

Can. J. Neurol. Sci. 1986; 13:511-516

From the Kinsmen Laboratory of Neurological Research, Department of Psychiatry, University of British Columbia and Department of Mathematics. Simon Fraser University, Vancouver

Reprint requests to: Dr. Edith G. McGeer, Kinsmen Laboratory of Neurological Research. Department of Psychiatry. University of British Columbia, 2255 Wesbrook Mall, Vancouver, B.C., Canada V6T IW5 
Alzheimer's disease (DAT) has classically been defined as a progressive dementia which is definitively diagnosed by the presence of numerous plaques and tangles in the brain, particularly in the hippocampus and some cortical regions. More recently, considerable evidence has been reported that DAT is also characterized by a deficiency in cortical cholinergic innervation' and in cortical glucose metabolism. ${ }^{2}$ Other biochemical abnormalities, including many neurotransmitter defects, have also been reported but so far the cholinergic and metabolic defects seem to be the ones best correlated with mental status. In this paper we will discuss some positron emission tomographic (PET) studies with ${ }^{18} \mathrm{~F}$-deoxyglucose (FDG) which support a correlation between defective cortical metabolism and mental status, present some autopsy data on one PET-scanned case of DAT which suggest that plaques and tangles may be a transient phenomena and, finally, show some acetylcholinesterase (AChE) histochemical results on autopsy brains which suggest that $\mathrm{AChE}$-positive fibers may frequently play an important role in the evolution of plaques.

\section{Methods}

PET was carried out on the UBC-TRIUMF PETT-VI high resolution system which yields data on 14 slices of brain, 7 millimetres apart, ${ }^{3}$ using in each case, approximately 5 millicuries of FDG. Data were collected as described elsewhere, ${ }^{3,4}$ and values for the local cerebral metabolic rate for glucose (LCMRgl) were determined, as previously described, for both large cortical and subcortical areas ${ }^{4}$ and smaller ( $5 \mathrm{~mm}$ square) areas in individual cortical gyri, ${ }^{5,6}$ using the postmortem brain and appropriate landmarks from the scans for precise anatomical localization. MRI was carried out on a Picker Vista cryogenic machine at a field strength of 0.15 Tesla using inversion recovery sequences. CT was performed on a Siemens Somatom DR2 whole body scanner. Care was taken in the positioning of patients to match the orientation in the PET studies. ${ }^{4}$

Demented cases were selected from among the patients referred to the Alzheimer's Clinic at the U.B.C. Health Sciences Center. Each had a complete physical and neurological examination and was given a battery of psychological tests by Dr. H. Tuokko ${ }^{4.7}$ or colleague. Elderly normal volunteers for the PET studies were given the same psychological tests.

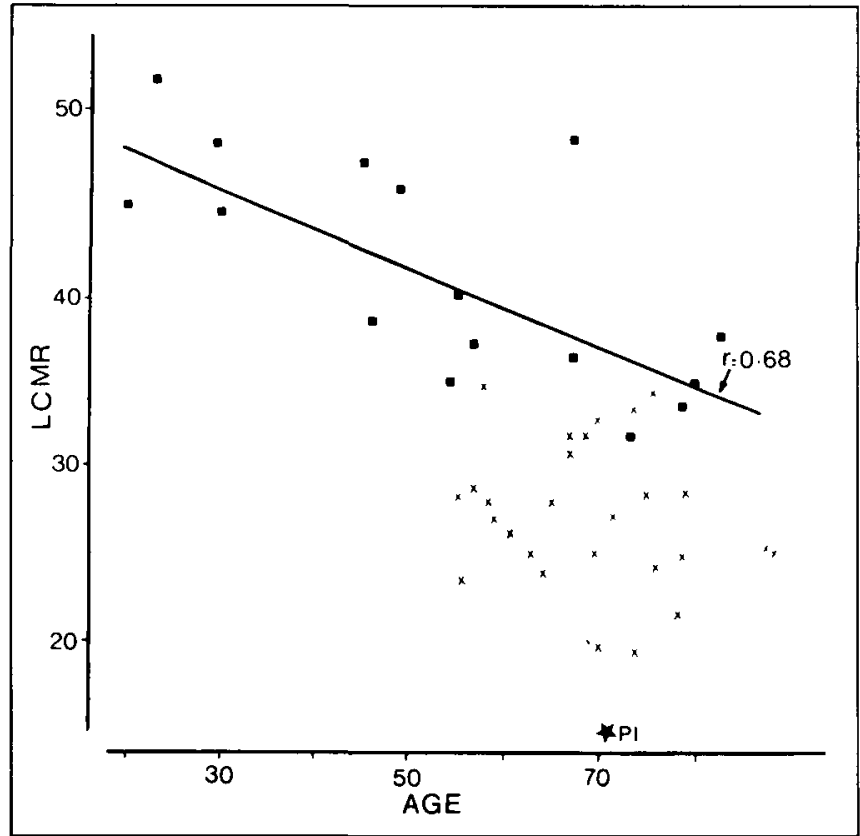

Figure 1 - Plotoflocal cerebralmetabolic ratefor glucose (LCMRgl)against age in the frontal cortex (average of left and right values) for a number of normals ( and line or correlation) and of clinically diagnosed Alzheimer's disease $(x)$. The value found in the single case of Pick's disease confirmed at autopsy is also shown (PI).

Two demented males who received PET scans have come to autopsy. One developed dementia at age 79 and had a typical DAT pattern on PET studies at age 82; he had been included in the series on which we initially reported. ${ }^{4} \mathrm{He}$ died at age 83 and the diagnosis was confirmed on autopsy. ${ }^{5}$ The other who had been scanned at age 73 , fifteen months before death and 5 years after first developing memory problems, had not been included in the reported series because the scan was highly atypical; on autopsy this proved to be a case of Pick's disease. ${ }^{6}$ Autopsies in these two cases were performed, respectively, at 15 and 2 hours post-mortem. A number of small cortical samples were taken for analysis of ChAT and the brains then fixed and sectioned for cresyl violet, immunohistochemical glial fibrillary acidic protein (GFA) and Bielschowsky's silver staining using standard techniques. More complete histories on these cases and on the experimental methods are published elsewhere. ${ }^{5.6}$

Table 1: Local cerebral metabolic rate for glucose as a percent of cerebellar activity in various brain regions in groups of 24 presumed Alzheimer's cases (age $69 \pm 8$ years) and controls ( $66 \pm 10$ years) and single cases of Alzheimer's disease (aged 83 years) and Pick's disease (aged 75 years) confirmed at autopsy.

\begin{tabular}{|c|c|c|c|c|c|c|}
\hline & \multirow[b]{3}{*}{ Controls } & \multirow{3}{*}{$\begin{array}{c}\text { Presumed } \\
\text { Alzheimer } \\
\text { Cases }\end{array}$} & \multicolumn{4}{|c|}{ Autopsy Confirmed Cases } \\
\hline & & & \multicolumn{2}{|c|}{ Alzheimer's } & \multicolumn{2}{|c|}{ Pick's } \\
\hline & & & Left & Right & Left & Right \\
\hline Parietal cortex & $107 \pm 14 \%$ & $89 \pm 16 \% \%^{* *}$ & $55 \% \%^{* *}$ & $88 \% *$ & $95 \%$ & $109 \%$ \\
\hline Occipital cortex & $115 \pm 10 \%$ & $103 \pm 15 \% *$ & $90 \% * *$ & $117 \%$ & $122 \%$ & $112 \%$ \\
\hline Frontal cortex & $106 \pm 16 \%$ & $90 \pm 23 \% *$ & $86 \%$ & $107 \%$ & $63 \% * *$ & $40 \% * *$ \\
\hline Thalamus & $108 \pm 15 \%$ & $107 \pm 7 \%$ & $101 \%$ & $103 \%$ & $94 \%$ & $77 \% * *$ \\
\hline Basal ganglia & $115 \pm 44 \%$ & $127 \pm 14 \%$ & $128 \%$ & $150 \%$ & $105 \%$ & $106 \%$ \\
\hline
\end{tabular}

'For each group the figure given is the mean \pm S.D. of the average of left and right and the significance of the difference between groups is indicated by ${ }^{* * *} \mathrm{p}<0.001 ;{ }^{* *} \mathrm{p}<0.01 ;{ }^{*} \mathrm{p}<0.05$. For the individual cases ** or * indicate, respectively, more than two or more than one standard deviation below control mean. 
Autopsy brains from 3 controls (average age 65.3, range 50 to 75 years) and 6 DAT cases (average age 75.8 , range 68 to 83 years) were used for the AChE histochemical studies reported here. The brains were fixed by perfusion and post-fixation, sectioned on a freezing microtome and stained by a new AChE method as previously described. ${ }^{8.9}$ Adjacent sections were stained for cresyl violet, for tangles and plaques by the modified Bielschowsky's method, or by the modified thioflavin T method for amyloid.

\section{RESULTS}

Analysis of the data on large ROIs in the neurologically normal controls indicated a significant decrease with age in most of the cortical regions (Figure 1). Hence the data on the presumed DAT cases were compared with those in a subgroup of normals of similar age. Statistical analysis indicated that the DAT group had mean LCMRgls significantly below the controls in several cortical regions (data not shown) and, when the data were "normalized" by expressing them as a percent of the cerebellar LCMRgl in the individual brain, the DAT group was low in the frontal, parietal, temporal and occipital cortices, but not in the thalamus, basal ganglia or precentral gyrus (Table 1). As previously reported, the DAT cases scanned ranged from mild to severely affected and the more impaired cases generally showed greater and more widespread metabolic deficits. ${ }^{4,5}$ The deficits did not correlate very well with atrophy as seen in $\mathrm{MRI} / \mathrm{CT}$ scans and the application of various methods of analysis indicated the low metabolic rates were due to poor tissue metabolism and not to inclusion of CSF space. ${ }^{4.5 .6}$
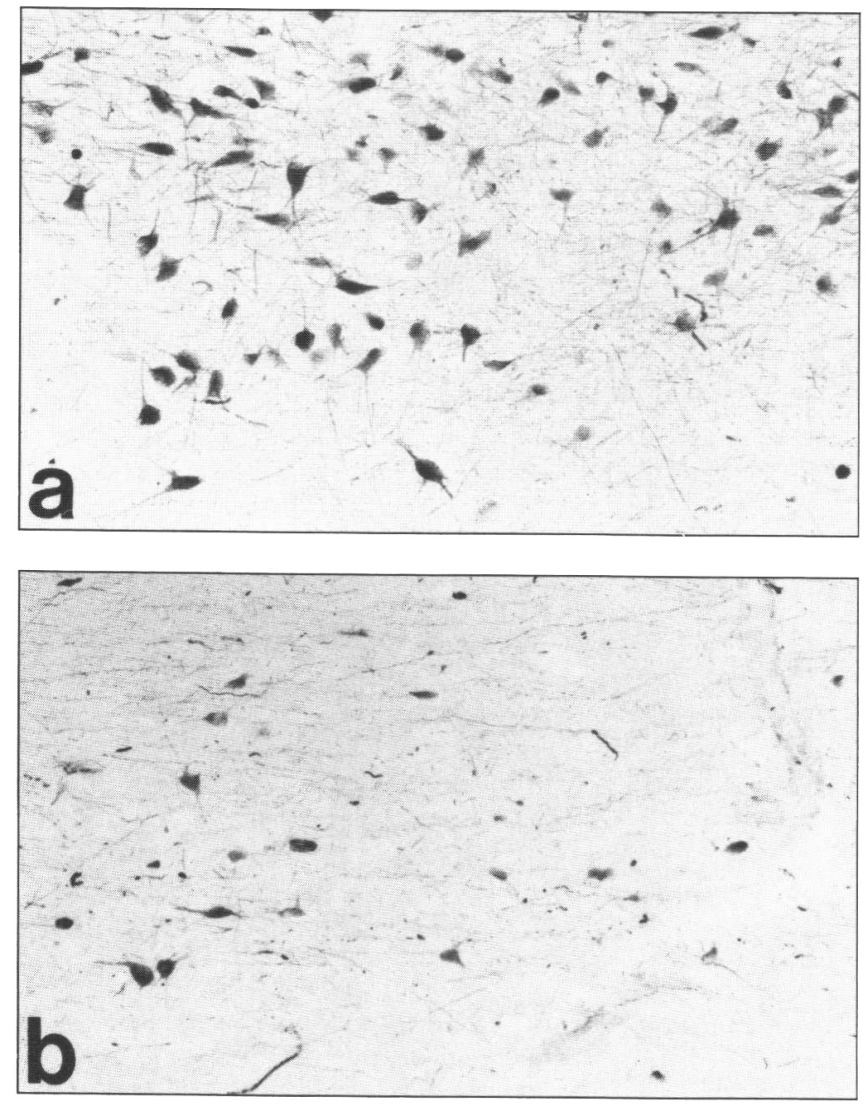

The DAT case which came to autopsy was particularly revealing in that there was a wide variation in the degree of pathological involvement in various areas of the cortex, thus permitting meaningful correlations to be made with in vivo imaging modalities. At autopsy the gross appearance of the brain correlated with MR and CT images which showed some regional atrophy. This was much less revealing than the PET images which correlated with microscopic findings of neuronal loss and proliferation of glia. In the PET scan done 15 months before death on this then severely affected individual, the LCMRgls were particularly low in the left parietal, temporal and occipital lobes. The large deficits in the LCMRgl in the left hemisphere were well beyond what could be accounted for by atrophy or partial volume effects. Instead they correlated with microscopic changes. The right occipital lobe, for example, was one of the least involved areas in the PET study, showing only a moderate metabolic defect in most gyri (75-84\% of control on absolute LCMRgl), and in this area there was still a definite laminar organization of the neurons although there were many senile plaques and some tangles, with nests of glia around the plaques being revealed by the GFA stain. This corresponds to mild to moderate or grade 1-2 degeneration as defined by Brun and Englund.$^{10}$ The left frontal cortex (where the absolute LCMRgl was 56-73\% of control) showed marked neuronal loss and disruption of the laminar cortical pattern, spongioform degeneration, and many plaques and tangles. GFA staining showed moderate gliosis in all layers of the cortex. This corresponds to grade 3 , or severe degeneration. ${ }^{10}$ The left parietal region, with a very severe metabolic defect (41-47\% of control) showed a
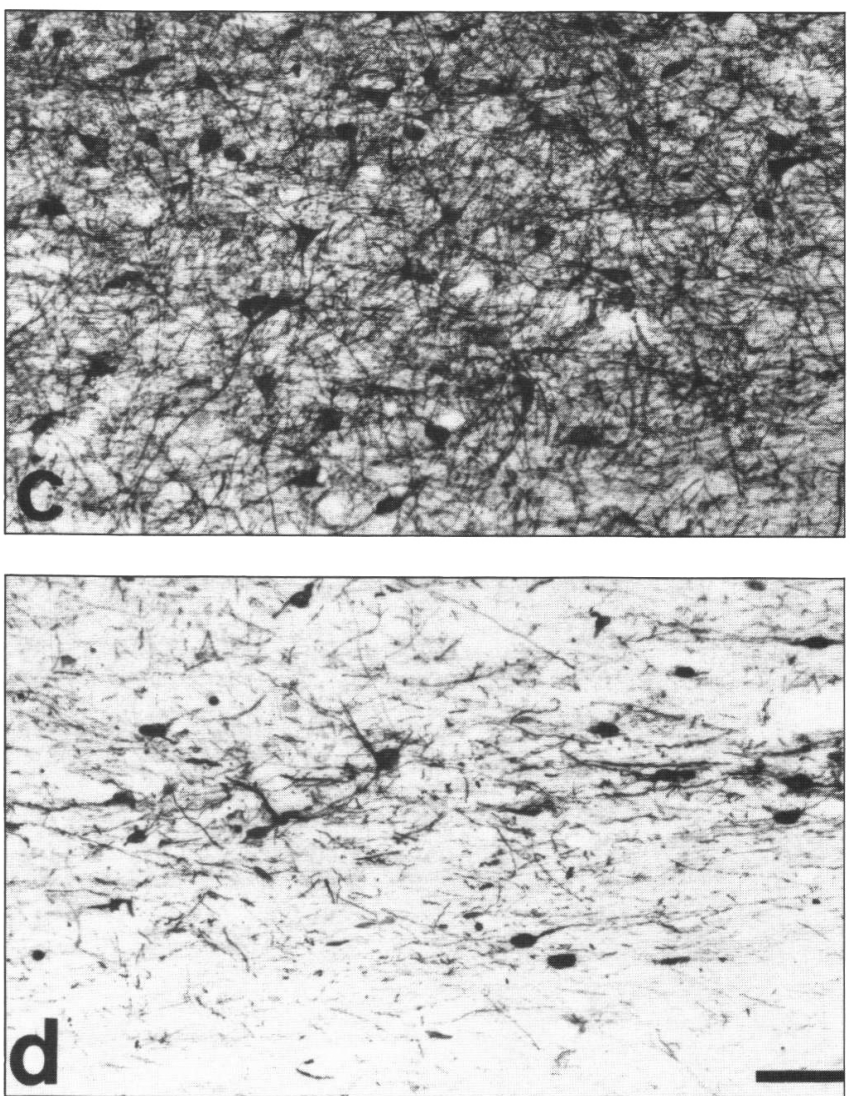

Figure 2 - Comparison of cholinergic structures in the substania innominata (SI) area of normal $(a, c)$ and Alzheimer's $(b, d)$ brains in sections stained immunohistochemically for choline acetyltransferase $(a, b)$ and histochemicals for acetylcholinesterase $(b, d)$. These comparative sections were taken at the same coronal levels just posterior to the anterior commissure $(B a r=100 \mu \mathrm{m})$. 
still different pattern. Here the cortical mantle was thin and the white matter had shrunk to produce gross atrophy. Microscopically, cresyl violet staining showed extensive astrocytosis, a very marked loss of neurons with some residue of the spongioform state, and no laminar pattern. There was intense GFA staining throughout the gray matter, but the frequency of plaques was less, presumably because of neuronal disappearance. This "burned out" stage of the disease corresponds to grade 4 , or maximal degeneration. ${ }^{10}$

The PET pattern on the case of Pick's disease which has come to autopsy was sufficiently distinctive to suggest that it might be possible to distinguish Pick's from Alzheimer's disease premortem. ${ }^{6}$ This case showed the lowest LCMRgl yet found in the frontal cortex (Figure 1) but the other cortical areas were relatively unaffected (Table 1). On autopsy, it was found that the specific gyri showing sharply decreased LCMRgls were those where there was extensive gliosis and neuronal loss. More moderate decreases were found in areas with numerous Pick bodies and inflated neurons but less gliosis. In order to test the hypothesis that low PET LCMRgl values correlated with areas showing the most profound gliosis as opposed to frequency of Pick bodies and inflated neurons, Spearman rank order correlation coefficients for the percentage decreases in LCMRgl vs. various indices of histological abnormalities were calculated for the 11 right cortical gyri on which both PET and histological data were available. These calculations supported the hypothesis since the correlation coefficients found were:
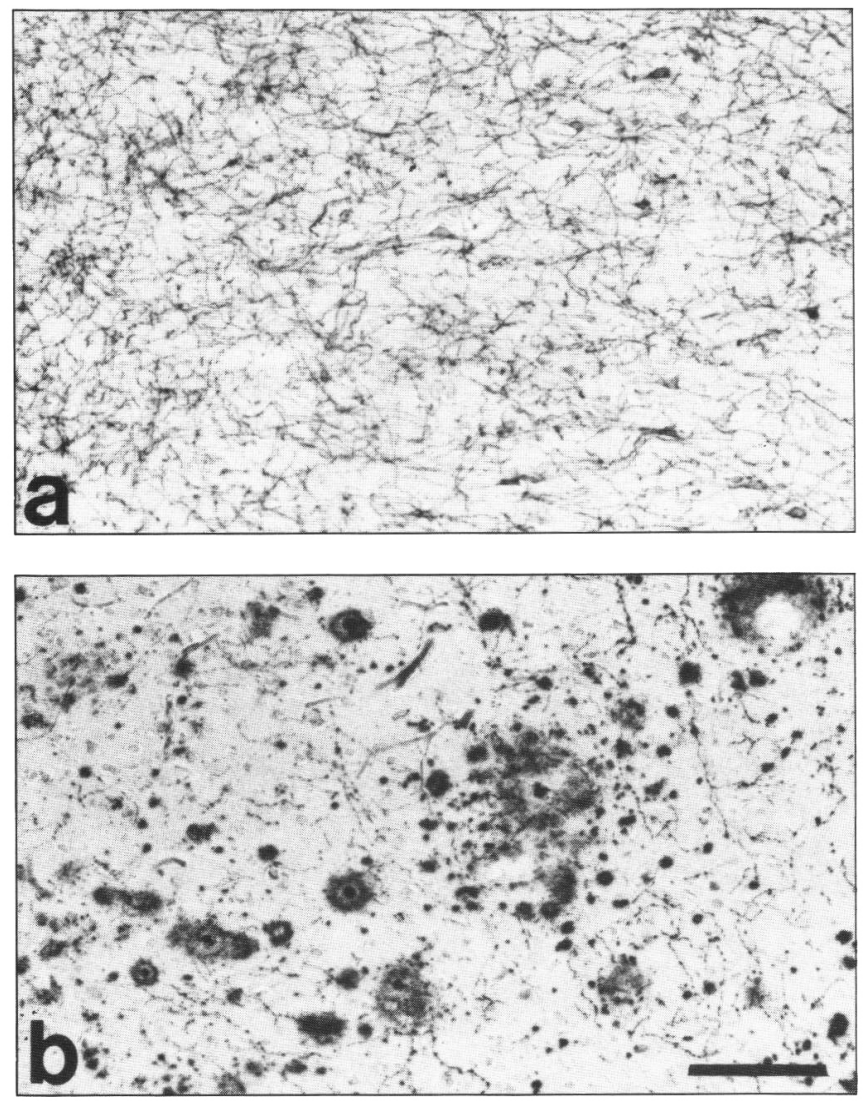

Figure 3 - AChE-positive fibers in comparable cortical sections from a normal (a) and an Alzheimer's (b) brain. There is extensive loss of AChE positive fibers in the Alzheimer's cortex and the residual fibers are often associated with plaque-like lesions $($ Bar $=100 \mu \mathrm{m})$ for metabolic decreases vs. gliosis ( 0.78$)$; vs. neuronal cell loss (0.57); vs. Pick bodies + inflated neurons (0.17); and vs. Pick bodies alone $(0.10){ }^{6}$

Results on ChAT on samples from most cortical gyri gave values in the normal range $(0.60-0.92 \mu \mathrm{mol} / \mathrm{h}-100 \mathrm{mg}$ protein) and clearly above those previously reported for Alzheimer's cases $\left(0.14-0.25 \mu \mathrm{m} / \mathrm{h}-100 \mathrm{mg}\right.$ protein). ${ }^{11}$ This finding is consistent with the normal substantia innominata (SI) cell count which was found. Tissue samples taken from areas of cortex that were severely atrophied, however, showed very low ChAT activities
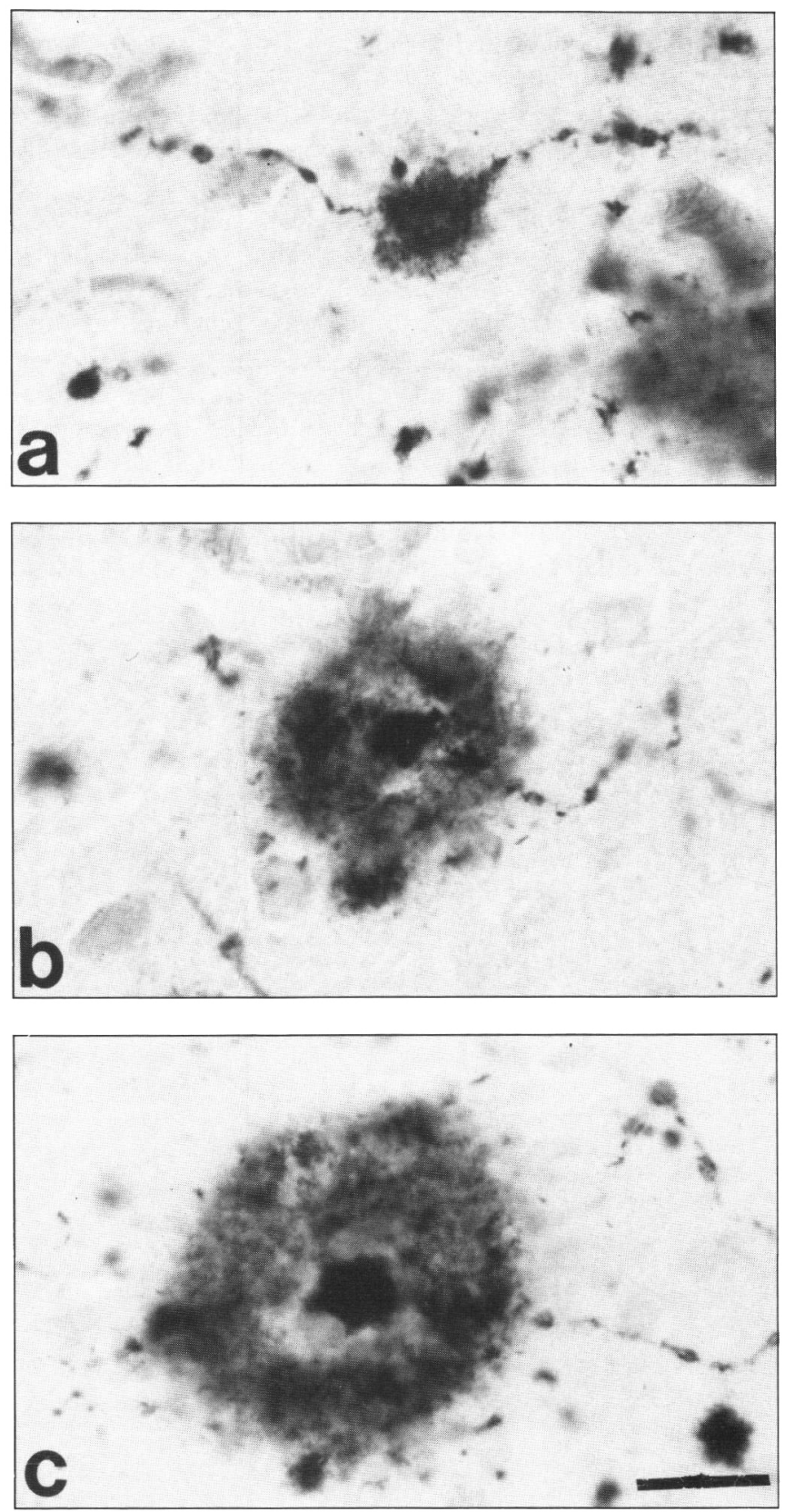

Figure 4-Various stages of AChE-positive plaques seen in the cortex in Alzheimer's brains. a) An AChE-positive fiber with several varicosities and $a$ small plaque. $b$ ) A larger plaque showing a more diffuse spread of AChE-positive material and emergence of a core. c) A prominent plaque with an AChE-positive core, a large and even more diffuse halo of $A C h E$-positive material and the remaining $A C h E$-positive fiber. All are at the same magnification $(B a r=20 \mu \mathrm{m})$. 
(0.02-0.09 $\mu \mathrm{m} / \mathrm{h}-100 \mathrm{mg}$ protein), indicating loss of nerve endings in these heavily gliosed regions.

In both ChAT immunohistochemical and $\mathrm{AChE}$ histochemical studies, all the DAT's cases showed, as expected, ${ }^{12}$ a substantial loss of cholinergic neurons in the SI as compared with controls (Figures 2a,b and 2c,d).

In the cortex, there was also a remarkable difference between normal and DAT's brains in AChE-positive structures. In control brains (Figure 3a), an extensive network of AChE-positive fibers could be seen. The fibers were thin, with numerous varicosities and frequent branches, but in the DAT cases only a few AChE-positive fibers remained (Figure $2 b$ ). The reduction in AChE-positive fibers in DAT cortex was highly similar to that seen in rat brain where the SI had been electrolytically lesioned. There was a major difference, however, in the appearance of the residual cortical AChE-positive fibers in the DAT cases compared with the lesioned rats. In DAT, many of the fibers showed randomly enlarged swellings, which, on adjacent sections stained with Bielschowsky's stain, were seen to be classical senile plaques. In the superficial layers of the cortex where cholinergic fibers terminate, several small AChE-positive plaques (5-30 $\mu \mathrm{m}$ in diameter) could often be seen aligned along a single fiber. AChE-positive plaques are illustrated in a series of photomicrographs in Figure 4. Figure 4a shows an AChEpositive cortical fiber with several small swellings, as well as an unmistakable plaque. Figure $4 \mathrm{~b}$ shows a more diffuse spread of $\mathrm{AChE}$-positive material from an enlarging plaque and the emergence of a core within the ragged outlines. Figure $4 \mathrm{c}$ shows a fiber with a prominent plaque consisting of an AChE-positive core with a slightly irregular surface surrounded by a more enlarged halo of AChE-positive material.

\section{Discussion}

Defective cortical metabolism in DAT has now been reported from a number of groups (for review see ${ }^{2}$ ). The DAT cases we have studied have been more mixed with regard to ages and disease severity than those used in most studies and we have found considerable variation from individual to individual in the specific cortical regions affected. Overall, however, there appeared to be good correlation with psychological deficits and this has been supported by longitudinal studies on several individual cases (not reported here). Availability of the correlative MRI/CT images and the use of a number of different analytical methods have allowed us to argue that the deficits seen reflected deficient tissue metabolism and not artefacts due to adventitious inclusion of metabolically inert CSF. This argument is supported by the correlative studies between postmortem histology and premortem PET data which indicate that the metabolic deficits correlate with neuronal loss and gliosis. These studies also indicate that the "characteristic pathologies", i.e. plaques and tangles in DAT and Pick's bodies in Pick's disease, may represent a transient phase between normal neuronal structure and complete neuronal degeneration with glial proliferation. The important difference between Pick's and DAT is the more confined nature of severe gliosis in the former. Pick's disease should therefore be suspected if sharp LCMRgl deficits appear which are confined to one or both frontal lobes or temporal lobes in the absence of CT or MRI evidence of a vascular lesion. This hypothesis can be verified as more demented cases, including those with disorders such as Huntington's disease and progressive supranuclear palsy, come to autopsy subsequent to PET scanning.

While the plaques in DAT may be transient, they are clearly an important phenomenon and the histochemical results using a new, highly sensitive method for $\mathrm{AChE}^{8.9}$ suggest the following hypothesis for the evolution of many senile plaques: 1) initially there are swellings along impaired $\mathrm{AChE}$ axons with, usually, multiple sites on a single axon; 2) AChE-positive material escapes from the membranes surrounding ballooning areas as they continue to enlarge; 3 ) repair processes reduce the AChE-positive discharge, producing an AChE-intense core but an expanding halo of previously discharged AChE-positive material; 4) the AChE-positive core consolidates but the halo continues to expand, becoming therefore more diffuse and even less AChE-positive compared with the core. AChE staining of plaques in Alzheimer's disease has been previously reported ${ }^{13}$ but with less detail than described here. The probable importance of cholinergic afferents to the cortex in the evolution of senile plaques has been suggested for both Alzheimer's disease ${ }^{14}$ and aged monkeys. ${ }^{15}$ Whether other neuronal systems are also involved remains to be determined.

\section{ACKNOWLEDGEMENTS}

This research was supported by grants from the Medical Research Council of Canada, the Mr. and Mrs. P.A. Woodward's Fdn., the B.C. Medical Services Fdn. and the Alzheimer's Support Association of B.C. It owes much to the Alzheimer's Clinic, UBC Health Sciences Center, and to the UBC/TRIUMF PET team. In particular, we acknowledge the help of Dr. M.J. Adam, Dr. W. Ammann, Dr. M. Ball, Dr. L. Beattie, Dr. D. Calne, Ms. M. Craig, Mrs. M. Coval, Ms. M. Durand, Dr. D.K.B. Li, Dr. W. Martin, Dr. B. Pate, Dr. J.G. Rogers, Dr. T.J. Ruth, Ms. C.I. Sayre, Ms. P. Schofield, Dr. J. Stoessel, Ms. J. Suzuki and Dr. H. Tuokko.

\section{REFERENCES}

1. Katzman R. Alzheimer's disease. New Engl J Med 1986; 314: 964-973.

2. McGeer PL. Brain imaging in Alzheimer's disease. Brit Med Bull 1986; 42: 24-28.

3. Martin WRW, Beckman JH, Calne DB, et al. Cerebral glucose metabolism in Parkinson's disease. Can J Neurol Sci 1984; 11: 169-173.

4. McGeer PL, Kamo H, Harrop R, et al. Positron emission imaging in clinically diagnosed Alzheimer's disease patients. Can Med Assoc J 1986; 134: 597-607.

5. McGeer PL, Kamo H, Harrop R, et al. Comparison of PET, MR and CT scanning with pathology in a proven case of Alzheimer's disease. Neurology (in press).

6. Kamo H, McGeer PL, Harrop R, et al. Positron emission tomography and histopathology in Pick's disease. Neurology (in press).

7. Coval M, Crockett D, Holliday S, et al. A multi-focus assessment scale for use with frail elderly populations. Can J Aging 1985; 4 : 101-109.

8. Tago H, Kimura T, Maeda $\mathrm{H}$. Detailed acetylcholinesterase fiber and neuronal staining in rat brain by a new and highly sensitive histochemical procedure. J Histochem Cytochem (in press).

9. Mizukawa K, McGeer PL, Tago H, et al. The cholinergic system of the human hindbrain studied by choline acetyltransferase immunohistochemistry and acetylcholinesterase histochemistry. Brain Res 1986; 379: 39-55.

10. Brun A, Englund E. Regional pattern of degeneration in Alzheimer's disease: neuronal loss and histopathological grading. Histopath 1981; 5: 549-564.

11. McGeer EG, Singh EA, McGeer PL. $y$-Glutamyl transferase: normal cortical levels in Alzheimer's disease. Alzheimer's Disease and Associated Disorders (in press). 
12. McGeer PL, McGeer EG, Suzuki J, et al. Aging, Alzheimer's disease and the cholinergic system of the basal forebrain. Neurology $1984 ; 34: 741-745$.

13. Friede RL. Enzyme histochemical studies of senile plaques. J Neuropath Exp Neurol 1965; 24: 477-491.
14. Arendt T, Bigl V, Tennstedt A, et al. Neuronal loss in different parts of the nucleus basalis is related to neuritic plaque formation in cortical target areas in Alzheimer's disease. Neuroscience 1985; 14: $1-14$.

15. Struble RG, Hedreen JC, Cork LC, et al. Acetylcholinesterase activity in senile plaques of aged macaques. Neurobiol Aging 1984; 5: 191-198. 